\title{
Imaginer-lire Le Capital
}

Das Kapital imaginieren-lesen

Imagining-reading The Capital

\section{Julien Pieron}

\section{OpenEdition}

\section{Journals}

Édition électronique

URL : http://journals.openedition.org/ceg/1221

DOI : $10.4000 /$ ceg. 1221

ISSN : 2605-8359

\section{Éditeur}

Presses Universitaires de Provence

Édition imprimée

Date de publication : 18 décembre 2015

Pagination : 165-174

ISBN : 979-1-03200-020-5

ISSN : 0751-4239

\section{Référence électronique}

Julien Pieron, « Imaginer-lire Le Capital », Cahiers d'Études Germaniques [En ligne], 69 | 2015, mis en ligne le 17 décembre 2017, consulté le 27 novembre 2020. URL : http://journals.openedition.org/ceg/ 1221 ; DOI : https://doi.org/10.4000/ceg.1221 


\title{
Imaginer-lire Le Capital
}

\author{
Julien PIERON
}

Université de Liège

Les pages qui suivent esquissent quelques réflexions sur le travail de Kluge, à partir du projet-fleuve que constituent les Nouvelles de l'Antiquité idéologique (Nachrichten aus der ideologischen Antike) ${ }^{1}$. Sous-titré Marx - Eisenstein - Le Capital, ce projet est constitué par un ensemble de trois DVD totalisant près de dix heures de matériel audio-visuel, accompagné d'un livret d'une soixantaine de pages et de trois séries d'histoires (Geschichten) extraites de précédents ouvrages de Kluge. En désignant la tentative de filmer le texte de Marx dans les pas d'Eisenstein par la formule « imaginer-lire Le Capital», on n'entend pas suggérer que Kluge se tromperait de texte, mais que le processus de lecture qu'il nous propose est inséparable d'une réflexion sur la mise en image - au double sens d'une présentation figurée et d'un travail de l'imagination.

Pour commencer à déplier le problème de la mise en image, on posera une question simple: qu'est-ce qui nous est donné à voir dans les Nouvelles de l'Antiquité idéologique? Paradoxalement, ce qui est donné à voir dans ces Nachrichten, ce sont d'abord des mots (écrits) et des paroles (parlées) - quantitativement bien plus de mots et de paroles que d'images au sens habituel du terme.

Des mots écrits, d'abord. Presque dès l'ouverture, et tout au long des 9h30 de film, le spectateur est confronté à de longs défilés d'intertitres grâce auxquels des notes d'Eisenstein ou des passages de Marx et d'autres auteurs (Nietzsche, Adorno, Korsch, Enzensberger) sont figurés, mis en images sur le mode du calligramme. Le texte, pourtant statique, semble danser en caractères et en couleurs contrastés; les mots montent, descendent, se tordent ou se décomposent dans un mouvement graphique visant à figurer le sens de ce qui est dit: lorsque la bourse s'effondre, ou que le navire d'une époque s'engloutit, le texte s'effondre, la phrase bascule du coin supérieur gauche au coin inférieur droit. Si ce procédé de figuration de l'esprit par la lettre peut être de prime abord déconcertant pour le spectateur-lecteur non germaniste, il se révèle, sur la durée, à la fois captivant et rassurant: le dessin des mots sur la page brise la monotonie tout en facilitant la saisie du sens.

Au-delà des mots écrits, ce sont aussi des paroles parlées qui sont données à voir. Une bonne partie des Nouvelles est en effet consacrée à exhiber - et tout d'abord à faire naître, dans un climat de liberté et de confiance - une parole gravitant autour

Alexander Kluge, Nachrichten aus der ideologischen Antike. Marx - Eisenstein - Das Kapital, Frankfurt am Main, Filmedition Suhrkamp, Suhrkamp Verlag, 2008. 
du texte de Marx. Parole d'interprètes, au double sens de " commentateurs » plus ou moins autorisés (on trouvera parmi ces interprètes aussi bien des universitaires qu'un romancier ou une traductrice, qui s'entretiennent avec un Kluge qu'on ne voit pas toujours, mais dont on sent constamment la présence amicale) et de " performeurs » qui donnent à entendre des textes de Marx. Divers dispositifs encadrent ces deux types d'interprétation. Kluge s'emploie à mettre en variation le décor où séjournent ses interprètes: studio de télévision, bureau-bibliothèque, salle de château, pièce obscure munie d'un éclairage d'un autre temps (ampoule électrique rudimentaire, lampe à huile ou à pétrole), fausse fenêtre sur laquelle défilent des ciné-collages ou des paysages urbains en accéléré. Le spectre de performances suscitées va de l'entretien, ou du simple acte de lire un texte " en costume de ville », à celui de le bonimenter sur le mode de la leçon de choses ou de le lire dans un contexte fortement théâtralisé, déguisements et maquillage à l'appui - comme dans la séquence intitulée «Le Marx des débuts et le Marx tardif» («Der frühe Marx und der späte Marx », DVD III, 6), où un bourgeois du XIX ${ }^{\mathrm{e}}$ siècle et un homme préhistorique lisent à haute voix l'incipit du livre I du Capital.

Ce qui ouvre les Nouvelles de l'Antiquité idéologique, ce n'est pourtant pas une simple lecture, mais la tentative ambitieuse de filmer les notes de travail d'Eisenstein relatives au projet, datant des années 1927-1929, de filmer le capital "d'après le scénario de Karl Marx » et suivant la manière de l'Ulysse de Joyce (DVD I, 1). Dans cette séquence magistrale se télescopent lecture, boniment, succession d'intertitres, montage d'images d'archive et d'extraits de films anciens retravaillés en vidéo (notamment des films d'Eisenstein, parfois postérieurs au projet d'adaptation du Capital), mosaïques mouvantes réalisées à partir d'un écran divisé en multitude de cases, travail sur l'environnement sonore. La présentation klugienne du projet jamais réalisé par Eisenstein semble alors coïncider avec la vision fugitive de son achèvement virtuel - comme dans les romans fondés sur le principe de la mise en abyme, où l'histoire du roman en train de se faire finit par constituer le roman lui-même.

Directement enchaîné à cette ouverture, l'entretien avec l'historienne Oksana Bulgakowa restitue la folie et le souffle d'un Eisenstein drogué, exténué par le travail de montage et de réduction de son film Octobre, presque aveugle, et qui n'envisage plus, comme unique tâche et seule issue possible à son travail sur les formes, que le projet de dépasser tout ce qui a été précédemment réalisé au cinéma en se confrontant à l'adaptation du Capital, "d'après le scénario de Karl Marx », et selon le procédé de l'Ulysse de Joyce, qu'il découvre au même moment (DVD I, 2). Ce procédé consiste selon Eisenstein à condenser, en suivant les mouvements d'un travailleur et de sa femme durant une demi-journée (midi-minuit), l'histoire entière de l'humanité, qu'il s'agira de déployer en montant des chaînes d'associations reliant les choses et les gestes, de la quotidienneté la plus triviale à l'histoire universelle telle qu'on peut la décoder à l'aide des outils forgés par Marx (fétichisme de la marchandise, lutte des classes, etc.). On passera ainsi de la soupe à l'affaire Dreyfus, ou de la morale sexuelle à la fabrication de la soie.

Ce que ce prologue jubilatoire pourrait laisser attendre, de prime abord, c'est une poursuite du projet jamais réalisé par Eisenstein. L'ensemble des entretiens 
menés par Kluge - qui constituent, en volume horaire, la partie la plus substantielle des Nouvelles de l'Antiquité idéologique - semblent d'ailleurs animés par ce désir de reprendre, sous d'autres latitudes sociales et temporelles, le projet d'Eisenstein. Presque toutes les interviews commencent par un résumé de la tentative d'Eisenstein et sont traversées par les questions : «Qu'est-ce qui, selon vous, intéressait Eisenstein dans le livre de Marx, et dans le rapprochement avec Joyce?»; « Que pourrait-on filmer ou mettre en image là-dedans, et comment? "; " Où sont les images dans Le Capital? »; ou encore: "Que pourrait-on inventer comme images pour mener à bien ce projet aujourd'hui?».

Contrairement à l'attente première, il semble pourtant que ces entretiens ne débouchent pas sur une réalisation audio-visuelle «totale » du type de celle qu'on trouvait dans la séquence d'ouverture, qu'ils n'aboutissent pas à l'accomplissement de l'œuvre dans la réflexion sur l'œuvre à faire. Avec une extrême brutalité, et en sautant immédiatement à la séquence de clôture, on pourrait presque dire qu'ils n'aboutissent à rien - qu'ils tournent en eau de boudin, parce qu'après neuf heures de recherche et d'expériences diverses, en lieu et place d'un point d'orgue qu'on attendrait un peu frappant ou virtuose, l'ensemble se clôt par une séquence grotesque, vrai faux entretien dans lequel l'acteur comique Helge Schneider alterne, durant plus d'une demi-heure, les rôles d'ouvrier au chômage qui suit des cours du soir de marxisme, d'acteur de cinéma engagé dans deux projets parallèles de film sur Karl Marx et Jim Morrison («Celui qui aura la meilleure musique sera le film principal », nous dit-on), et de compositeur russe fantaisiste proposant, sur un synthétiseur pourri, des solutions d'orchestration ainsi qu'une série d'images kitsch ou grotesques pour achever de façon spectaculaire mais néanmoins heureuse (happy end) le projet d'Eisenstein (DVD III, 16).

Il semble donc qu'il faille faire machine arrière, revenir sur l'ensemble des séquences enchaînées par Kluge, et concevoir d'une autre façon le travail des Nouvelles de l'Antiquité idéologique. Pour ce faire, il convient de se laisser porter par quelques-unes des pistes dégagées dans les entretiens.

1. Le premier grand entretien sur Marx, entre Kluge et Hans Magnus Enzensberger, en vient à aborder des questions d'éducation (DVD I, 15). Comment se fait-il qu'il reste parfois si peu de ce qu'on a passé des années à apprendre sur les bancs de l'école? Comment se fait-il que des gens qui ont été abreuvés de philosophie marxiste ne soient pas en état de l'utiliser et de la réactualiser pour penser la crise financière qui fait rage au moment où sont réalisées les Nouvelles (2008)? Enzensberger répond en substance que l'oreille de l'enfant est sensible au ton, qu'elle détecte immédiatement le ton faux, se ferme au rabâché ou au pétrifié, et que le texte de Marx est en quelque sorte trop précieux pour être enseigné, qu'il doit plutôt être lu en cachette sous la table. Il apparaît alors que les lectures de Marx qui ont précédé, accompagnées non pas de musique emphatique, mais le plus souvent d'airs très calmes et paisibles (notamment des transcriptions au piano de la Norma de Bellini), doivent d'abord être considérées comme autant de recherches de ce ton juste, décapé de tout rabâchage ou pétrification. Il n'est d'ailleurs pas anodin que l'entretien avec Enzensberger prenne place dans une séquence située sous le signe de la fluidification du pétrifié, et se poursuive par un dialogue au cours duquel Kluge 
joue à se faire traduire et commenter - de l'allemand vers le russe et du russe vers l'allemand - des fragments du texte de Marx.

2. Deuxième piste: l'entretien avec Peter Sloterdijk, traversé par l'idée qu'il faut lire Le Capital plutôt comme un texte poétique que comme une analyse scientifique (DVD II, 4). Au-delà d'une critique du positivisme ou du scientisme marxiste, cette proposition de lecture, introduite par diverses références à l'Antiquité (Ulysse et ses épithètes homériques de polymètis, polymèchanos; Ovide et les Métamorphoses), se décline selon trois motifs. Le premier motif fondamental qui gouverne l'entretien est celui d'une pluralité des types d'intelligence: Sloterdijk y oppose l'esprit de sérieux (qui produit des savants et des croyants) à l'esprit de ruse (incarné dans les figures du sophiste, de l'avocat, du trickster, et dont Ulysse est une des incarnations paradigmatiques). Ce qui singularise ce second type d'intelligence, c'est que tout y devient effet, jeu, stratégie. Les paroles ne sont plus conçues comme des constats, mais comme des actes de langage, des mouvements au sein d'une joute dont chaque acteur cherche à être aussi le metteur en scène. À l'instar des sophistes, Marx aurait donc une conscience aiguë et une véritable pratique de la dimension pragmatique du discours. Si l'auteur du Capital est un metteur en scène, on comprend que la réflexion sur la forme, la figuration et la mise en image ne soit pas un exercice secondaire mais une façon de dialoguer avec lui en touchant au cœur même de sa démarche.

Le deuxième motif fondamental de l'entretien avec Sloterdijk est l'importance de la métamorphose, envisagée non comme un simple déguisement, mais comme une transformation autrement profonde, qui advient quand il n'est plus d'autre issue possible. Ce motif, qui traverse tout l'entretien, est introduit par la question rituelle: «Qu'est-ce qui intéressait Eisenstein dans l'Ulysse de Joyce? Pourquoi rapprocher Ulysse et Le Capital? ». Sloterdijk répond par l'idée d'un mouvement circulaire, d'un voyage et d'un retour d'Ulysse-Bloom/de l'argent, couplé à une série de métamorphoses et à la difficulté de reprendre sa forme initiale. Il affirme également qu'il conviendrait d'interdire la lecture du Capital isolément et d'ajouter au canon Marx-Engels-Lénine l'Ovide des Métamorphoses. Ce parallèle Marx/Ovide fait émerger le thème du Märchen, de l'affinité entre l'esprit du conte - cher à Benjamin et l'intelligence accordée à la lecture du Capital. Ce que propose Sloterdijk, c'est au fond de relire Marx de façon "symétrique » (au sens du principe de symétrie de David Bloor, ou plutôt de Bruno Latour), c'est-à-dire en prenant au sérieux - et même en amplifiant - les outils critiques forgés par Marx, tout en les appliquant au texte marxien lui-même, non pour le retourner ou le démolir, mais pour l'accentuer de manière nouvelle.

Cette proposition peut s'illustrer par la discussion du fétichisme, qui constitue un troisième motif fondamental de l'entretien. On comprend habituellement la théorie du fétichisme comme un outil critique de démystification du monde social. Sloterdijk retravaille la notion, en prenant à la lettre ce qui fonctionnait de manière encore imagée ou métaphorique dans le texte de Marx. Pour comprendre le fétichisme, il faut revenir au fétiche, dans une perspective anthropologique: mettre l'accent non sur ce qu'il est (une telle posture nous ferait passer à côté du mode d'existence du fétiche), mais s'intéresser à la façon dont il est patiemment fabriqué - dont il en vient à vivre, mais aussi à mourir, à survivre et à hanter. Sloterdijk décrit le lent 
processus de fabrication du fétiche, dans lequel on plante des clous en le gonflant petit à petit d'affects, de sentiments humains, de malédictions ou de bénédictions ${ }^{2}$, puis conclut que ce mouvement de fabrication du fétiche correspond tout à fait à la façon dont Marx envisageait le devenir de son propre texte, qui circule en se chargeant petit à petit de toutes les colères, révoltes et aspirations humaines. Se pose ensuite la question du fétiche comme objet guerrier: à supposer qu'on veuille refuser la façon dont certains fétiches vivent et agissent parmi nous, il ne suffit pas de mettre au jour le processus de leur fabrication (les forces accumulées en eux le sont réellement et ne s'évaporent pas dans une simple prise de conscience), il faut en quelque sorte les désamorcer, déminer patiemment le terrain miné par la lente accumulation des affects et leur donner la possibilité de s'agencer différemment. La nouvelle accentuation de la catégorie de fétiche permet donc de garder la compréhension génétique ou généalogique de l'état-des-choses-et-des-sentiments, mais en rend la sortie beaucoup plus lente, difficile et problématique.

On notera - et le fait est assez représentatif de l'espèce de discours indirect libre que les Nouvelles tissent progressivement entre Kluge et ses interprètes - que la direction indiquée par cette reprise hétérodoxe de la théorie du fétichisme déborde largement le cadre de l'entretien avec Sloterdijk. On la retrouve dans le courtmétrage «Der Mensch im Ding » réalisé par Tom Tykwer, qui travaille davantage dans le sens d'une micro-histoire des choses - dont la coprésence à la moindre action humaine est soulignée et comme démultipliée - que dans celui d'une analyse structurelle de leur mode de production (DVD II, 1), dans la séquence consacrée au « Lamento des marchandises invendues » (DVD I, 8), ainsi que dans de nombreuses interventions de Joseph Vogl autour de concepts fondamentaux de la critique marxiste tels qu'idéologie ou aliénation. Elle apparaît aussi dans la façon dont Kluge soumet à Vogl la proposition de disserter sur l'idée d'une vitalité des choses et d'un droit-de-l'homme des choses («Gibt es ein Menschenrecht der Dinge? ») - ce qui entraîne Vogl à introduire les idées d'agency et même de parlement des choses (DVD III, 14). La volonté de lire Marx comme un poète ou un raconteur témoigne donc de l'animisme assumé d'un Kluge dont le projet global d'investiguer le « côté subjectif » (« subjektive Seite ») du Capital vise tout autant les choses que l'homme.

3. La troisième piste essentielle à la compréhension de la démarche de Kluge est l'entretien avec Oskar Negt, autour de la question de savoir comment lire Le Capital (DVD III, 9). Au fil de sa conversation avec Kluge, Negt en vient à atteindre un point de perplexité: finalement, pourquoi filmer Le Capital plutôt que la Critique de la raison pure ou La Science de la logique? Les images qu'on peut extraire du Capital ne sont-elles pas fondamentalement insuffisantes, impuissantes à rendre le cadre théorique dans lequel réside précisément la force de l'analyse marxiste? Du point de vue des images, une chaumière brûlée est une chaumière brûlée; l'analyse de Marx permet pourtant de comprendre en quoi une chaumière brûlée sous Élisabeth $\mathrm{I}^{\mathrm{re}}$ diffère toto caelo d'une chaumière brûlée sous Gengis

2 À partir de l'image de clous plantés dans une statue de Hindenburg, Kluge saisit l'occasion d'établir un rapprochement entre le monde africain et le monde occidental - nouvelle façon de symétriser le propos et d'éviter l'exotisme. 
Khan. En ce point de perplexité, la balance se renverse: Negt devient pensif, Kluge commence par faire des propositions d'images incongrues (la terre vue du ciel la nuit, et la concentration des points lumineux dans certaines régions comme image du zèle et de la coopération), avant de souligner combien l'illustration visuelle « littérale» d'images rencontrées dans Le Capital (par exemple le travail des enfants dans les mines ou les fabriques, dont on voit défiler des vignettes) tend à souligner la distance qui nous sépare du texte. Là où Negt insistait sur la pertinence et l'actualité des analyses de Marx, Kluge propose une mise à distance du texte marxien, une façon de faire de Marx l'équivalent d'un historien grec ou romain - qui ne nous donne pas des outils immédiatement applicables dans le présent, mais doit permettre par contraste une distanciation de notre expérience présente ( De l'utilité de l'Antiquité: distanciation de l'expérience », «Vom Nutzen der Antike: Verfremdung der Erfahrung », affirme un intertitre), à la faveur de laquelle on peut recommencer à l'imaginer («Cela doit être aussi éloigné que la lune, de sorte que je puisse à nouveau le peupler par mon imagination »). Illustrer de façon littérale le texte de Marx, donner à voir les images qu'il contient, reviendrait donc non pas à le rendre plus proche, mais au contraire à l'éloigner.

C'est en ce sens d'un éloignement ou d'une mise à distance que peuvent également être compris les choix musicaux qui accompagnent la lecture des textes de Marx : la musique de Bellini ou de Verdi est aussi celle du monde - définitivement révolu - dans lequel Le Capital a vu le jour. Le projet de faire de Marx un auteur antique, clairement affirmé dans le livret d'accompagnement, apparaît aussi dans le découpage du premier DVD: sa deuxième section, qui donne à entendre une série de textes marxiens lus sur une calme musique d'opéra, porte le titre « Nostalgie de l'enfance des pensées » ( Sehnsucht nach der Kindheit der Gedanken »). Le texte célèbre de l'Introduction de 1857 - sur le paradoxe d'un art grec qui nous émeut encore, alors même que ses conditions matérielles d'émergence ont définitivement sombré dans le passé, comme une enfance historique qui ne reviendra pas ${ }^{3}$ - y est en quelque sorte mis en abyme par Kluge: la lecture émerveillée qu'en fait la comédienne Sophie Rois, et le dispositif dans lequel s'insère cette lecture, font que ce texte ne désigne plus notre rapport à l'art grec, mais notre rapport au texte de Marx lui-même (DVD I, 11).

\footnotetext{
« Mais la difficulté n'est pas de comprendre que l'art grec et l'épopée sont liés à certaines formes du développement social. La difficulté, la voici: ils nous procurent encore une jouissance artistique, et à certains égards, ils servent de norme, ils nous sont un modèle inaccessible. Un homme ne peut redevenir enfant sans être puéril. Mais ne se réjouit-il pas de la naïveté de l'enfant, et ne doit-il pas lui-même s'efforcer, à un niveau plus élevé, de reproduire sa vérité ? Est-ce que, dans la nature enfantine, ne revit pas le caractère de chaque époque, dans sa vérité naturelle? Pourquoi l'enfance historique de l'humanité, au plus beau de son épanouissement, n'exercerait-elle pas l'attrait éternel du moment qui ne reviendra plus? Il est des enfants mal élevés, et des enfants précoces. Beaucoup de peuples de l'Antiquité appartiennent à ces catégories. Des enfants normaux, voilà ce que furent les Grecs. Le charme que nous trouvons à leurs œuvres d'art n'est pas contrarié par le peu d'avancement de la société où elles ont fleuri. Il en est plutôt le résultat; il est inséparable de la pensée que l'état d'immaturité sociale où cet art est né, où seul il pouvait naître, ne reviendra jamais. ", in Karl Marx, Introduction générale à la critique de l'économie politique, in Euvres, I, Économie I, Paris, Gallimard (coll. « Bibliothèque de la Pléiade »), 1994 [1857], p. 266.
} 
Couplé aux réflexions qui précèdent, le désir de faire de Marx un auteur antique, et le paradoxe d'une figuration sensible du concept rendant un mode de pensée non pas plus proche mais au contraire plus distant, permet de pointer la coexistence de différents régimes de mise en image au sein des Nouvelles. On en distinguera au moins trois.

1. Un régime d'illustration didactique, présentant des images parfaitement accordées au discours: vignettes, vieilles photos, peintures, chromos. L'effet paradoxal de ces images serait qu'elles mettent à distance plutôt qu'elles ne rapprochent, créent un décrochage ou une distanciation du présent sur fond de passé, et permettent par cette béance même une colonisation du présent par l'imagination.

2. Un régime - déceptif - d'absence d'illustration: les longs entretiens filmés, qui, au lieu d'en exposer didactiquement les lignes de force théoriques, creusent les textes canoniques du marxisme, y introduisent du jeu et des inflexions nouvelles, mais ne donnent lieu à aucune illustration immédiate - sinon une illustration ponctuelle, purement informative et didactique, selon le régime précédemment évoqué (parler d'Ovide ou de Staline, et montrer leur portrait). Cette mise en image contrariée, cette déception d'une attente d'image, peut elle aussi être conçue comme un appel à l'imagination: non pas donner à lire Le Capital, mais le donner à lire et à imaginer - à imaginer-lire - à travers une série d'actes de parole (dialogue, traduction, jeu, parodie) qui libèrent le texte en le pluralisant, et apparaissent comme autant de vecteurs possibles de métamorphose et de fluidification du pétrifié.

3. Un régime de figuration d'une théorie ou d'un concept encore informulé. C'est peut-être sur ce mode que fonctionnent quelques-unes des séquences les plus intéressantes des Nouvelles: la série de natures mortes industrielles projetées en accéléré, mais aussi les séquences réalisées en collaboration avec Helge Schneider. Tous ces passages offrent des images dont on ne sait pas immédiatement ce qu'elles figurent au juste, de quel enjeu théorique défini elles sont l'image. Par là, elles fonctionnent comme autant d'appels à un travail de glose et au déploiement de l'imagination conceptuelle du spectateur - bref à ce que Kant nommait « jugement réfléchissant », vecteur de création conceptuelle pour des intuitions encore sans nom.

Ainsi la séquence intitulée «Soll/Ist 》- du nom d'un des ponts aux ânes de la philosophie morale - donne-t-elle à voir en accéléré le travail sur une chaîne de montage automobile; les deux mentions «Soll» et « Ist», suivies chacune d'un nombre en constante variation, figurent sur un tableau comptabilisant les voitures à assembler et celles qui sont déjà montées (DVD I, 4). Face à ce genre d'image, qui ne peut être directement réintégrée dans un cadre théorique ou un dispositif d'agitprop, la pensée reste comme en suspens. Le travail à la chaîne n'y apparaît pas d'emblée comme une forme de vie insupportable; la grande opposition philosophique entre être et devoir-être est ramenée à une dimension matérielle élémentaire, sans qu'on sache au juste qu'en faire, quelle conséquence en tirer d'un point de vue théorique. Le même suspens apparaît face aux «paysages avec industrie lourde classique » («Landschaft mit klassischer Schwerindustrie ») filmés à la façon de natures mortes dont le défilement accéléré accentue l'importance de la fumée et des cieux changeants, donnant à voir - et à entendre - quelque chose comme le grondement de l'immobilité (DVD I, 5). Le même suspens se produit enfin dans les séquences où 
intervient Helge Schneider - séquences visiblement bouffonnes, dont les costumes outrés (le bleu de travail avec clé à molette débordant d'une poche, la barbe postiche démesurée, etc.) attestent clairement le côté artificiel, mais dont les répliques d'apparence souvent absurdes sont avancées de façon étonnamment réaliste.

Penchons-nous sur l'une de ces séquences, classée dans la rubrique « extras »: «Le travailleur collectif devant Verdun» («Der Gesamtarbeiter vor Verdun», DVD II, 6). Cette séquence se présente comme un montage de textes et d'images suivi d'une vraie fausse interview de "Zigarren-Willi », maître-artificier durant la guerre de 14-18. L'entretien nous apprend que deux équipes de soldats-mineurs allemands et français passent leur temps à creuser les collines du village de Vauquois et à y placer des charges d'explosifs - le but de la manœuvre étant d'approcher le plus près possible du tunnel de l'ennemi et de le dynamiter par en dessous. Le maitre-artificier décrit avec beaucoup de professionnalisme son activité, confesse son admiration pour le savoir-faire de l'ennemi, et déploie une morale fondée sur le goût du travail bien fait et le culte d'une habileté qui constitue pour le travailleur sa patrie véritable. Dans le livret accompagnant le DVD (p. 36), Kluge ajoute que du point de vue d'un observateur extraterrestre, le grand ballet des artificiers français et allemands pourrait apparaître comme l'exemple même de la coopération - coopération dans laquelle, du point de vue terrestre, les parties prenantes travaillent pourtant à leur anéantissement réciproque. Une fois glosée par Kluge, cette séquence, qui laisse d'abord songeur, peut apparaître rétrospectivement comme mise en image originale du texte de Marx, au sens où elle contient à la fois les idées classiques de division du travail et d'aliénation, tout en y ajoutant une série d'éléments relevant de ce que Kluge nomme le « côté subjectif »- l'économie affective du Capital en nous.

On clôturera cette réflexion par l'évocation d'une dernière séquence, qui présente la particularité d'inclure en son sein le travail de glose permettant à l'image « réfléchissante » de devenir figuration d'une pensée qu'elle contribue à faire naitre. Intitulée «Le monument et le vrai tombeau ( (Das Denkmal und das wahre Grab »), la séquence suit, caméra à l'épaule, un des bénévoles qui entretiennent le cimetière de Highgate à Londres, à la recherche de la « vraie tombe » ou du « vrai tombeau » de Marx (DVD II, 3). Kluge s'y saisit d'une découverte apparemment fortuite (l'existence de deux tombes, complètement différentes), pour la transformer en allégorie de deux réceptions ou destins posthumes de l'œuvre de Marx. D'un côté, le monument imposant commandé par les Russes, avec son bronze à grosse barbe et son " Prolétaires de tous les pays... » gravé dans le marbre. De l'autre, une modeste dalle de pierre, brisée et rongée par les ans. Cette dalle, située dans un coin perdu du cimetière parce que Marx était juif, le bénévole peinera à la localiser et devra plusieurs fois télé-communiquer pour la retrouver. Juste avant que la pierre soit donnée à voir, la séquence est suspendue par un entretien, au cours duquel Sophie Kluge est amenée à commenter, à la lueur d'une faible ampoule qui donne à la scène l'éclairage d'une peinture de Georges de La Tour, les images rapportées de Londres. Il y est question du mouvement et de l'immobilité des morts. Kluge suggère que le bénévole prend soin des morts; la jeune femme rectifie: il prend soin des chemins entre les morts. Apparaît alors l'image de la vraie tombe, qui surprend par sa pauvreté, sa nudité, et fait jaillir l'opposition entre un Marx massif, imposant, 
canonique ou canonisé, et un Marx brisé, multiple, encore et toujours secret. À la faveur de l'interruption pensive créée par le dialogue, la tombe brisée peut devenir figuration de la multiplicité de l'héritage - l'un des fils rouges de l'entreprise de Kluge - et de la possibilité d'un retour presque spectral.

En guise de conclusion, on proposera d'ajouter à celle de Kluge une autre version des deux tombes: celle de Mallarmé dans Le Tombeau d'Edgard Poe ${ }^{4}$.

Tel qu'en lui-même enfin l'éternité le change,

Le Poëte suscite avec un glaive nu

Son siècle épouvanté de n'avoir pas connu

Que la mort triomphait dans cette voix étrange!

Eux, comme un vil sursaut d'hydre oyant jadis l'ange

Donner un sens plus pur aux mots de la tribu

Proclamèrent très haut le sortilège bu

Dans le flot sans honneur de quelque noir mélange.

Du sol et de la nue hostiles, ô grief!

Si notre idée avec ne sculpte un bas-relief

Dont la tombe de Poe éblouissante s'orne

Calme bloc ici-bas chu d'un désastre obscur

Que ce granit du moins montre à jamais sa borne

Aux noirs vols du Blasphème épars dans le futur.

L'intérêt et la force de ce poème est qu'il thématise ce déploiement de l'imagination que les divers régimes de figuration pratiqués par Kluge dans les Nouvelles semblent avoir pour but de provoquer. Comme la séquence sur « Le monument et le vrai tombeau », le poème de Mallarmé n'est rien d'autre que le commentaire ou la glose poétique d'un objet trouvé - d'où l'importance du scolie rédigé par Mallarmé pour l'édition Deman de ses Poésies, qui nous apprend que le «calme bloc » existe bel et bien, et que c'est à partir de lui que le poète travaille. Le monument de Poe à Baltimore y est en effet présenté comme «un bloc de basalte que l'Amérique appuya sur l'ombre légère du poëte, pour sa sécurité qu'elle n'en ressortît jamais ${ }^{5}$ ». $\mathrm{Au}$ danger que le monument ne soit qu'une façon d'exorciser les spectres et de barrer le chemin des morts vers les vivants, Mallarmé répond par un acte de langage - le poème doit être lu lors de l'inauguration du monument, c'est au sens le plus haut un texte de circonstance - qui est aussi appel à la puissance de l'imagination. L'enjeu est de renverser le rapport des forces en présence, et de conférer une autre efficace au monument: l'injonction poétique métamorphose le bloc-couvercle posé par une Amérique apeurée en protection du mort contre le blasphème des vivants (vers 13 et 14), tout en ouvrant la possibilité qu'une fois retravaillé par l'imagination d'un peuple de lecteurs qui y sculpteront de nouvelles images (vers 10 et 11), la vraie

4 Stéphane Mallarmé, Poésies, Paris, Gallimard (coll. «Poésie/Gallimard »), 2003, p. 60.

5 Ibid., p. 74: «Le Tombeau d'Edgard Poe - Mêlé au cérémonial, il y fut récité, en l'érection d'un monument de Poe, à Baltimore, un bloc de basalte que l'Amérique appuya sur l'ombre légère du poëte, pour sa sécurité qu'elle n'en ressortît jamais ». 
tombe - toujours menaçante, car poreuse - puisse surgir à même le monument. Ce que le poème prend donc pour thème, tout en appelant à son exercice et à son prolongement par un peuple encore manquant, c'est au fond le geste même qu'il commence à accomplir, et que tout le travail de Kluge sur l'héritage de Marx semble destiné à encourager: s'emparer du donné à l'aide d'une imagination poétique qui, par le jeu de ses gloses, le métamorphosera en y ré-ouvrant du possible. 